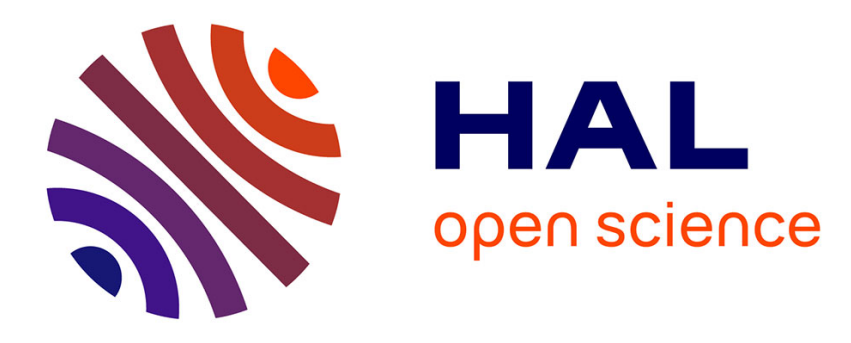

\title{
Weakly charged polyelectrolytes in a poor solvent
}

\author{
J.-F. Joanny, L. Leibler
}

\section{To cite this version:}

J.-F. Joanny, L. Leibler. Weakly charged polyelectrolytes in a poor solvent. Journal de Physique, 1990, 51 (6), pp.545-557. 10.1051/jphys:01990005106054500 . jpa-00212389

\section{HAL Id: jpa-00212389 https://hal.science/jpa-00212389}

Submitted on 1 Jan 1990

HAL is a multi-disciplinary open access archive for the deposit and dissemination of scientific research documents, whether they are published or not. The documents may come from teaching and research institutions in France or abroad, or from public or private research centers.
L'archive ouverte pluridisciplinaire HAL, est destinée au dépôt et à la diffusion de documents scientifiques de niveau recherche, publiés ou non, émanant des établissements d'enseignement et de recherche français ou étrangers, des laboratoires publics ou privés. 
Classification

Physics Abstracts

$36.20-61.40 \mathrm{~K}-61.10 \mathrm{E}$

\title{
Weakly charged polyelectrolytes in a poor solvent
}

\author{
J. F. Joanny $\left({ }^{1}\right)$ and L. Leibler $\left({ }^{2}\right)$ \\ $\left({ }^{1}\right)$ Institut Charles Sadron, 6 rue Boussaingault, 67083 Strasbourg Cedex, France \\ $\left({ }^{2}\right)$ E.S.P.C.I., 10 rue Vauquelin, 75005 Paris, France
}

(Reçu le 5 octobre 1989, accepté le 23 novembre 1989)

\begin{abstract}
Résumé. - Nous montrons qu'un polyélectrolyte faiblement chargé en mauvais solvant en l'absence de sel forme des mésophases lorsque la température est abaissée. Nous décrivons la transition d'apparition de ces mésophases à basse température en étudiant la possibilité de formation de micelles et à plus haute température en étudiant la divergence du pic à vecteur d'onde fini de la fonction de corrélation polymère-polymère. Si l'on ajoute du sel à la solution, le pic du facteur de structure disparaît et la transition devient une démixtion usuelle entre polymère et solvant. Le point multicritique associé à ces deux transitions est un point de Lifshitz; nous montrons par un critère de Ginzbourg que au voisinage de ce point les fluctuations de concentration jouent un rôle important et que la théorie de champ moyen n'est pas suffisante. A force ionique plus importante, la transition de démixtion appartient à la classe d'universalité d'Ising. Finalement, nous étudions brièvement une solution contenant des polymères faiblement chargés et des polymères neutres : le pic du facteur de structure disparaît lorsque la concentration en monomères neutres est augmentée.
\end{abstract}

\begin{abstract}
We show that a weakly charged polyelectrolyte in a salt free poor solvent undergoes a mesophase separation transition by lowering the temperature. This transition is investigated at low temperature by studying the possibility of micelle formation and at higher temperature by studying the divergence of the peak at finite wave vector of the polymer-polymer correlation function. As salt is added to the solution, the peak in the structure factor disappears and the transition becomes a usual demixing phase separation between polymer and solvent. The multicritical point of these two transitions is a so-called Lifshitz point ; in the vicinity of this point a Ginzburg criterion shows that concentration fluctuations play an important role and that the mean field theory is not sufficient. At higher salt concentration the demixing transition belongs to the Ising universality class. Finally, we discuss the behavior of solutions containing both weakly charged and neutral polymers : here also the peak in the structure factor disappears as the concentration of neutral polymers is increased.
\end{abstract}

\section{Introduction.}

The physical properties of neutral polymers both in dilute and semidilute solutions are well understood in terms of scaling laws. This very elegant approach seems however less successful when applied to electrically charged polymers and except for very dilute solutions where the 
chains take a « rodlike » configuration, our understanding of polyelectrolyte solutions is rather poor [1-5]. This is partly due to the long range character of the electrostatic interactions but also to the fact that polyelectrolyte solutions are even in the absence of salt ternary systems containing the solvent (generally water), the polyions and the counterions insuring the electrical neutrality: in fact the counterions are known to dominate many of the properties of the solution such as its osmotic pressure and may mask the macromolecular properties [6].

Another general feature of polyelectrolytes (a typical example being polystyrene sulfonate) is that they are organic polymers not soluble in water to which charged groups have been added : in the absence of the charged groups, the equivalent neutral polymer (polystyrene) would be below its $\theta$ point in a poor solvent ; the solution would not be stable and the polymer would phase separate from the solvent. The stability of the polyelectrolyte solution is thus only due to the existence of the ionic groups.

If only a small fraction $f$ of the monomers carries an ionic group, the solubility of the polymer is reduced because of the attractive interactions between neutral monomers and one may induce a demixing phase separation between polymer and solvent by lowering temperature.

In this paper, we discuss the nature of this phase separation for weakly charged polyelectrolytes where the fraction $f$ of charged monomers is small. We will require however that $f$ is sufficiently large for the electrostatic interactions to be relevant. This condition can be made more quantitative by considering a Gaussian polyelectrolyte with $N$ monomers and radius $R \sim N^{1 / 2} a$ : if a fraction $f$ of the monomers are charged the electrostatic energy of the chain is of order $(f N)^{2} \frac{\ell T}{R}$ where $\ell$ is the Bjerrum length characterizing the strength of the electrostatic interaction $\ell=\frac{e^{2}}{4 \pi \varepsilon T}=7 \AA$ in water $(e$ is the electronic charge and $\varepsilon$ the solvent dielectric constant); the electrostatic interaction is relevant if this energy is larger than the thermal excitation $T$. We thus demand

$$
N^{-3 / 4} \ll f\left(\frac{\ell}{a}\right)^{1 / 2} \ll 1
$$

Very dilute solutions of weakly charged polyelectrolytes have been studied by Khokhlov [7] who discussed the collapse of isolated chains. We focus here on more concentrated but still dilute solutions where the phase separation occurs and show that it is not a macroscopic phase separation between a polymer rich phase and a solvent rich phase but rather a mesophase formation, the mesophase consisting in polymer dense and polymer dilute regions arranged in a periodic array : the instability of the solution does not occur at zero wave vector but rather at a finite wave vector that fixes the period of the mesophase. Above the transition, the polymer structure factor $S(q)$ has a peak at a finite wave vector $q^{*}$; the peak diverges at the mesophase separation transition [8]. Such mesophase formation in polyelectrolyte solutions has already been recently suggested by Borue and Erukhimovich [9].

Qualitatively, the formation of mesophases may be understood from the following argument : the polymer has a tendency to aggregate and to form dense regions, if these dense regions are macroscopic, they must be electrically neutral and the entropy of the counterions is rather low; in the mesophase, the electroneutrality may be violated locally and the counterions can occupy the dilute phase around a dense polymer region, this considerably increases their entropy and lowers the free energy of the mesophase.

We first study the mesophase formation at low temperature by investigating the possibility 
of micelle formation and then discuss the high temperature limit where the transition is revealed by the divergence of the peak of the polymer-polymer concentration correlations.

As salt is added to the solution, the electrostatic interactions are screened and the peak in the structure factor disappears if the inverse Debye-Hückel screening length $\kappa$ becomes larger than the peak position: the mesophase separation transition becomes a usual macroscopic phase separation between polymer and solvent. The multicritical point associated with the change of the nature of the phase transition is a Lifshitz point. In section 3 , we study the behavior of the solution in the vicinity of this point in particular by estimating the importance of the concentration fluctuations. At higher ionic strength, we also discuss the possibility of a Fisher renormalization of the critical exponents of the consolute point.

Another way to eliminate the peak in the structure factor is to add to the polyelectrolyte solution a small amount of neutral polymer. At very low neutral polymer concentration the mesophase separation can still occur, the neutral polymer swelling the mesophase; as this concentration is increased, the polymer precipitates macroscopically from the solvent. This is discussed briefly in section 4 .

In the conclusion, we mention possible extensions of this work in particular to solutions in a good solvent and polyelectrolyte gels and discuss the relevance of our results to experiments.

\section{Mesophase separation transition.}

2.1 Micelle formation AT LOW TEMPERATURE. - We consider a very dilute salt free polyelectrolyte solution and follow here the approach of Khokhlov [7]. The temperature is lower than the $\theta$ temperature of the equivalent neutral polymer and is measured by $\tau=\frac{\theta-T}{\theta}$.

If the polymer forms a dense collapsed phase, the structure of this phase may be described in terms of closepacked thermal blobs [10]. As for neutral polymers, the size of the thermal blobs $\xi_{t}$, the number of monomers per blob $g_{t}$ and the local concentration are

$$
\xi_{\mathrm{t}}=a \tau^{-1}, \quad g_{\mathrm{t}}=\tau^{-2}, \quad \phi=\frac{\tau}{a^{3}} .
$$

Isolated chains in the solution are collapsed and in the limit of low charge fraction $f$ their free energy is dominated by the interfacial free energy between polymer and solvent. The radius of the chains is $R=\left(\frac{N}{g_{\mathrm{t}}}\right)^{1 / 3} \xi_{\mathrm{t}} \sim N^{1 / 3} \tau^{-1 / 3} a$ and the relevant interfacial tension is $\gamma \sim \frac{T}{\xi_{\mathrm{t}}^{2}} \sim T \tau^{2} a^{-2}$.

The chemical potential of these chains when the average monomer concentration is $\phi_{\mathrm{b}}$ reads

$$
\frac{\mu_{\mathrm{ex}}}{T}=(1+f N) \log \phi_{\mathrm{b}}+N^{2 / 3} \tau^{4 / 3}
$$

this expression including the translational entropy of both the polyion and the associated counterions and the interfacial free energy.

We now suppose that a micelle of $p$ chains and radius $R_{\mathrm{M}}$ forms and we discuss its stability [11]. The grand canonical free energy of the micelle is

$$
\frac{\Omega}{T}=\frac{\gamma}{T} 4 \pi R_{\mathrm{M}}^{2}+\alpha(p f N)^{2} \frac{1}{R_{\mathrm{M}}}-\frac{\mu_{\mathrm{ex}}}{T} p
$$


The first term is the interfacial free energy, the second term is the electrostatic free energy of the micelle where $\alpha$ is a numerical constant of order $1(\alpha=1 / 2$ if all charges are on the surface, $\alpha=3 / 5$ if the charges are randomly distributed inside the micelle). This free energy neglects the elastic energy of the polymer chain but it can be directly checked a posteriori that this is never larger than the electrostatic term if the Bjerrum length is of the order of the monomer size a.

The concentration inside the micelle is given by equation (2) and $p N=\phi \frac{4 \pi}{3} R_{\mathrm{M}}^{3}$. At thermodynamic equilibrium, the free energy $\Omega$ is minimum and $\frac{\mathrm{d} \Omega}{\mathrm{d} p}=0$. The micelle is stable only if at the minimum the free energy is negative. The critical micelle concentration is thus defined as the point where, both $\Omega$ and $\frac{\mathrm{d} \Omega}{\mathrm{d} p}$ vanish

$$
\log \phi_{\mathrm{cmc}} \sim-\frac{N^{-1 / 3} \tau^{4 / 3}}{f} \text {. }
$$

The size of the micelle at the c.m.c. decreases with the fraction of charged monomers

$$
R_{\mathrm{M}} \sim\left(\frac{a^{4}}{\ell f^{2}}\right)^{1 / 3} .
$$

The total number of chains in the micelle is then

$$
p \sim \frac{a \tau}{N f^{2} \ell}
$$

The micelle exists only if it contains more than one chain i.e. if $f<\left(\frac{\tau}{N}\right)^{1 / 2}$. If $f$ is larger than $\left(\frac{\tau}{N}\right)^{1 / 2}$, the micelle is smaller than one chain and each chain makes a small cigar made by several aligned micelles as discussed by Khokhlov [7].

Another necessary condition for micelles to exist is that their size be larger than the thermal correlation length $\xi_{\mathrm{t}}$. This leads to an estimate of the upper temperature where micelle formation occurs

$$
\tau \sim f^{2 / 3}\left(\frac{\ell}{a}\right)^{1 / 3}
$$

This very crude model allows only for the formation of spherical micelles; as the concentration is increased above the c.m.c., other mesophases may become more stable than spherical micelles : wormlike micelles, lamellar phases... We hope to discuss their relative stability in a future work.

The addition of a monovalent salt to the solution at a concentration $n$ screens the electrostatic interactions over a Debye-Hückel length $\kappa^{-1}$ such that $\kappa^{2}=8 \pi n \ell$. If the Debye length is larger than the micelle size $\left(\kappa R_{\mathrm{M}} \ll 1\right)$, the effect of screening is small and the characteristics of the micelles are given by equations $(6,7)$. If the screening length is smaller than the micelle size $\left(\kappa R_{\mathrm{M}} \gg 1\right)$, the micelles are no longer stabilized by their electrostatic energy and the polyions behave as neutral polymers with a slightly increased $\theta$ temperature $\frac{\theta^{\prime}-\theta}{\theta} \sim f^{2} /(\kappa a)^{2}$ (due to the electrostatic excluded volume between charged monomers) 
showing thus a macroscopic phase separation from the solvent. The maximum salt concentration for which micelle formation occurs is

$$
n \sim f^{4 / 3} a^{-8 / 3} \ell^{-1 / 3}
$$

2.2 MESOPHASE FORMATION AT HIGH TEMPERATURE. - At higher temperatures, the modelisation of the mesophase by dense and dilute regions separated by a sharp interface certainly breaks down, the width of the interface being of the order of the mesophase period. We there discuss mesophase formation by studying the stability of the homogeneous high temperature phase. In general, the solution contains the monomers at a concentration $\phi$, the counterions at a concentration $\phi_{i}$ and some salt at a concentration $n$. For simplicity, we assume that the polyion and the salt have the same counterion. The overall neutrality of the solution imposes that the charge density $\rho=e\left(f \phi+n-\phi_{\mathrm{i}}\right)$ vanishes.

The free energy of the solution may be written in a Flory manner

$$
\frac{F}{T}=\frac{\phi}{N} \log \phi+\phi_{\mathrm{i}} \log \phi_{\mathrm{i}}+n \log n+\frac{1}{2} v \phi^{2}+\frac{1}{6} w^{2} \phi^{3} .
$$

This assumes that the small ions are pointlike and form an ideal solution. The second virial coefficient between monomers (charged or neutral) $v=-a^{3} \tau$ is negative in a poor solvent and the third virial coefficient $w^{2}$ is of order $a^{6}$. The free energy does not include any electrostatic contribution, the classical polarization effects of electrolyte solutions [12] is negligible in this case.

The free energy associated with a fluctuation of the concentrations at a wave vector $q\left(\delta \phi_{q}, \delta \phi_{\mathrm{i} q}, \delta n_{q}\right)$ may be written as

$$
\frac{\delta F_{q}}{T}=\frac{1}{2}\left\{\delta \phi_{q}^{2}\left[S_{0}^{-1}(q)+v+w^{2} \phi\right]+\delta \phi_{\mathrm{i} q}^{2} \frac{1}{\phi_{\mathrm{i}}}+\delta n_{q}^{2} \frac{1}{n}\right\}+\frac{\delta F_{\mathrm{e} \ell}(q)}{T}
$$

$S_{0}(q)$ is the structure factor of an isolated polymer. In a poor solvent the polyelectrolyte chains are not stretched and $S_{0}(q)$ is essentially the structure factor of a Gaussian chain given by the Debye function; separating the contribution at zero wave vector, we write $S_{0}^{-1}(q)=\frac{1}{N \phi}\left[1+g\left(\frac{N q^{2} a^{2}}{6}\right)\right]$.

The fluctuations of concentration in general violate locally the electroneutrality and cost an electrostatic energy $\delta F_{\mathrm{e} \ell}=\frac{1}{2} \varepsilon E^{2}$ where $E$ is the local electric field. Using the Maxwell equation $i q E_{q}=\frac{1}{\varepsilon}\left(f \delta \phi_{q}+\delta n_{q}-\delta \phi_{\mathrm{i} q}\right)$, the electrostatic energy may be written as

$$
\frac{\delta F_{\mathrm{e} \ell}(q)}{T}=\frac{1}{2} \frac{4 \pi \ell}{q^{2}}\left(f \delta \phi_{q}+\delta n_{q}-\delta \phi_{\mathrm{i} q}\right)^{2} .
$$

This electrostatic energy prevents the charge fluctuations from becoming too important even at a finite wave vector.

Starting from the free energy (11) and using the equipartition of energy, one can calculate the relevant correlation functions (charge-charge, charge-monomer and monomer-monomer). We focus here on the monomer-monomer correlation function :

$$
S(q)=\left\langle\delta \phi_{q} \delta \phi_{q}\right\rangle=\frac{q^{2}+\kappa^{2}}{\kappa^{2} D+q^{2} D^{\prime}+\frac{q^{2}+\kappa^{2}}{N \phi} g\left(\frac{N q^{2} a^{2}}{6}\right)}
$$


$\kappa$ is the usual Debye-Hückel screening length to which only the salt and the counterions contribute $\kappa^{2}=4 \pi \ell\left(n+\phi_{i}\right)$. Notice that this is in general different from the screening length of the electrostatic interaction that may be calculated from the range of the chargecharge correlation function $\left\langle\delta \rho_{q} \delta \rho_{q}\right\rangle$.

$D^{\prime}=\frac{1}{N \phi}+v+w^{2} \phi$ is the inverse osmotic compressibility of the equivalent neutral polymer solution (with $f=0$ ). The spinodal line of the neutral polymer in the same solvent is given by $D^{\prime}=0$.

$D=D^{\prime}+\frac{f^{2}}{n+\phi_{\mathrm{i}}}$ is the inverse osmotic compressibility of the solution.

If the salt concentration is small enough, the correlation function $S(q)$ has a peak at a finite wave vector $q^{*}$ such that

$$
\left(q^{* 2}+\kappa^{2}\right)^{2}=\frac{24 \pi \ell f^{2} \phi}{g^{\prime} a^{2}}
$$

where $g^{\prime}$ is the derivative of $g\left(\frac{N q^{2} a^{2}}{6}\right)$ that takes the value $\frac{1}{2}$ if $\left(\frac{N q^{* 2} a^{2}}{6}\right)$ is large and $\frac{1}{3}$ if it is small.

A similar result was already proposed by Borue and Erukhimovich [9].

As the salt concentration is increased, $\kappa^{2}$ is increased and the peak shifts towards lower values. When $\kappa$ is larger than $\left(\frac{24 \pi \ell f^{2} \phi}{g^{\prime} a^{2}}\right)^{1 / 4}$ the peak disappears and $S(q)$ decays monotonically to zero.

As the temperature is lowered, the peak intensity diverges on the spinodal surface given by

$$
\frac{1}{N}+v \phi+w^{2} \phi^{2}+\frac{g\left(\frac{N q^{* 2} a^{2}}{6}\right)}{N}+\frac{g^{\prime} a^{2}}{6}\left(\frac{24 \pi \ell f^{2} \phi}{g^{\prime} a^{2}}\right)^{1 / 2}=0 .
$$

The instability at finite wave vector of the free energy indicates as said in reference [9] the formation of mesophases with a period $\frac{2 \pi}{q^{*}}$. The precize geometry of the phase diagram and the actual symmetry of the phases is obtained from a more refined analysis of the free energy including higher order terms and will be presented elsewhere. Notice that the more complete theory of instability at a finite wave vector first due to Brazovskii and extended to mesophase formation in block copolymers by Fredrickson predicts a change in the nature of the mesophase transition which is weakly first order even at a mean field critical point.

The temperature and concentration at the maximum of the spinodal line corresponding to the upper temperature where mesophase formation occurs is obtained from (15) as

$$
\tau_{\mathrm{c}} \sim f^{2 / 3}\left(\frac{l}{a}\right)^{1 / 3}, \quad \phi_{\mathrm{c}} \sim a^{-3} f^{2 / 3}\left(\frac{\ell}{a}\right)^{1 / 3} .
$$

This is in good agreement with the low temperature estimate of the previous section and crosses over smoothly to the consolute point of the polymer solvent mixture $\tau_{c} \sim N^{-1 / 2}$, $\phi_{\mathrm{c}} \sim N^{-1 / 2}$ in the limit where the charges have a negligible effect $\left(f<N^{-3 / 4}\right)$.

If the salt concentration is sufficiently large to eliminate the peak, the first instability of the free energy occurs at zero wave vector and corresponds to a usual demixing transition of a neutral polymer in a poor solvent with a slightly renormalized $\theta$ temperature as discussed in the previous section. We discuss this transition with more details in the following section. 


\section{Critical behavior in the vicinity of the Lifshitz point.}

At low salt concentration, the structure factor shows a peak that diverges at the mesophase separation transition; at higher ionic strength, the structure factor decreases monotonically and its value at zero wave vector diverges at the demixing transition. At a given ionic strength, each of these transitions has a critical point, the two critical points merge at a multicritical point called the Lifshitz point $[13,14]$.

3.1 POSITION OF THE LIFSHITZ POINT. - We first fix the salt concentration (or equivalently the inverse Debye-Hückel screening length $\kappa$ ) at a value higher than that of the Lifshitz point so that the solution undergoes a usual demixing transition. The consolute point is determined by using standard thermodynamics for three component (solvent-polymer-salt) solutions.

The spinodal condition indicating the locus of the points where the structure factor $S(q=0)$ diverges at zero wave vector is given by equation (13)

$$
D=\frac{1}{N \phi}+v+w^{2} \phi+\frac{4 \pi \ell f^{2}}{\kappa^{2}}=0 .
$$

The second condition giving the position of the consolute point on this line is given by the zero of a Gibbs determinant corresponding to the zero of the third derivative of the free energy for binary mixtures

$$
-\frac{1}{N \phi^{2}}-\frac{f^{4}(4 \pi \ell)^{3}}{\kappa^{6}} \phi+w^{2}=0 .
$$

The Lifshitz point is reached at the salt concentration where this consolute point belongs to the spinodal line of the mesophase transition with a peak at a wave vector $q^{*}=0$. Equation (14) gives

$$
\kappa^{4}=\frac{24 \pi \ell f^{2} \phi}{g^{\prime} a^{2}}
$$

Two regimes should be distinguished according to the value of $f N^{1 / 4}$ :

- if $f N^{1 / 4}$ is smaller than $\frac{w^{3 / 2}}{(4 \pi \ell)^{3 / 2} a^{3}}$ the coordinates of the Lifshitz point are

$$
\phi_{\mathrm{L}}=\frac{1}{N^{1 / 2} w}, \quad v_{\mathrm{L}}=-\left(\frac{4 \pi \ell}{18}\right)^{1 / 2} w^{1 / 2} f N^{1 / 4} a, \quad \kappa_{\mathrm{L}}^{4}=\frac{72 \pi \ell f^{2}}{N^{1 / 2} w a^{2}}
$$

- if $f N^{1 / 4}$ is larger than $\frac{w^{3 / 2}}{(4 \pi \ell)^{3 / 2} a^{3}}$

$$
\phi_{\mathrm{L}}=\frac{a^{6}(4 \pi \ell)^{3} f^{2}}{18^{3} w^{4}}, \quad v_{\mathrm{L}}=-\frac{18 w^{2}}{a^{2}(4 \pi \ell)}, \quad \kappa_{\mathrm{L}}^{4}=\frac{a^{4}(4 \pi \ell)^{4} f^{4}}{18^{2} w^{4}}
$$

3.2 Critical fluctuations. - The Flory theory used above is a mean field theory and is expected to give a poor description of the properties of the solution near critical points where concentration fluctuations become important. In order to test the validity of this theory in the vicinity of the Lifshitz point, we build now a Ginzburg criterion. We choose the salt concentration exactly at the Lifshitz point $\kappa=\kappa_{\mathrm{L}}$ and we compare the fluctuations of concentration $\left\langle\delta \phi^{2}\right\rangle$ at a temperature $v=v_{\mathrm{L}}+\varepsilon$ in the homogeneous phase $(\varepsilon>0)$ to the 
difference $\left(\phi_{+}-\phi_{-}\right)^{2}$ of the monomer concentrations in the two equilibrium phases at a temperature $v=v_{\mathrm{L}}-\varepsilon$.

The expansion of the Flory free energy (10) in the vicinity of the Lifshitz point gives

$$
\left(\phi_{+}-\phi_{-}\right)^{2}=N^{-1 / 2} \frac{\varepsilon}{w^{3}} \text {. }
$$

The fluctuations of monomer concentrations $\left\langle\delta \phi^{2}\right\rangle$ may be determined from the structure factor $S(q)$ given by equation (13) that we approximate by

$$
S(q)=\frac{\varepsilon^{-1}}{1+q^{4} \xi^{4}}
$$

where the correlation length $\xi$ diverges as

$$
\xi=\left(\frac{\kappa^{6} \varepsilon}{4 \pi \ell f^{2}}\right)^{-1 / 4} .
$$

In a mean field theory, the correlation length exponent is thus equal to $1 / 4$ at a Lifshitz point [12].

The average concentration fluctuation in a $d$-dimensional space is then estimated as

$$
\left\langle\delta \phi^{2}\right\rangle=\frac{1}{\xi^{d}} S(q=0) \sim \varepsilon^{-1}\left(\frac{\kappa^{6} \varepsilon}{4 \pi \ell f^{2}}\right)^{-d / 4} .
$$

This leads to a Ginzburg ratio

$$
X=\frac{\left\langle\delta \phi^{2}\right\rangle}{\left(\phi_{+}-\phi_{-}\right)^{2}} \sim \varepsilon^{d / 4-2} N^{1 / 2} \kappa^{3 d / 2} f^{-d / 2} .
$$

We recover here the well known result [12] that the upper critical dimension at which mean field starts to be valid is 8 for a Lifshitz point. Below 8 dimensions, concentration fluctuations are small and mean field theory is a good approximation if $X \ll 1$ i.e. in three dimensions if

$$
\begin{aligned}
& \frac{\varepsilon N^{1 / 2}}{w}>\left(f N^{3 / 4}\right)^{3 / 5} \gg 1 \quad \text { if } \quad f N^{1 / 4} \ll \frac{w^{3 / 2}}{(4 \pi \ell)^{3 / 2} a^{3}} \\
& \frac{\varepsilon N^{1 / 2}}{w}>N^{9 / 10} f^{12 / 5} \gg 1 \quad \text { if } \quad f N^{1 / 4} \gg \frac{w^{3 / 2}}{(4 \pi \ell)^{3 / 2} a^{3}} .
\end{aligned}
$$

In all cases there exists a significant range of temperature around the Lifshitz point where the mean field theory is not valid and thus a more refined theory is necessary [13].

3.3 Critical behavior AT higher Salt CONCENTRATion. - At high ionic strength the solution undergoes a usual demixing transition by lowering the temperature; at the consolute point, the critical behavior should be Ising-like as for neutral polymer solutions in a poor solvent. However, the position of the critical point depends on a conserved variable, the salt concentration. The salt acts thus as an impurity on usual binary mixtures : the presence of the salt may induce a renormalization of the critical exponents first discussed by Fisher [15]. The Ising exponents would be measured in an experiment where the salt chemical potential is maintained constant, in the real experiment where the ionic strength (the salt concentration) is constant, the exponents are renormalized in the very vicinity of the consolute point. In 
order to estimate the relevance of this renormalization, we construct a criterion analogous to the Ginzburg criterion and first introduced by Anisimov et al. [16].

In the experiment where the salt chemical potential is maintained constant, the grand canonical free energy has the usual Ising scaling behavior.

$$
A=A_{0}\left[\frac{v-v_{c}(\mu)}{v_{c}(\mu)}\right]^{2-\alpha}
$$

where $v_{\mathrm{c}}(\mu)$ is the critical value at fixed chemical potential $\mu$ of the second virial coefficient $v$ (equivalent to the temperature). Real experiments are performed at fixed salt concentration $n$ and the scaling variable is $\left[\frac{v-v_{\mathrm{c}}(n)}{v_{\mathrm{c}}(n)}\right]$. The relation between these two scaling variables is

$$
\left[\frac{v-v_{\mathrm{c}}(\mu)}{v_{\mathrm{c}}(\mu)}\right]=\left[\frac{v-v_{\mathrm{c}}(n)}{v_{\mathrm{c}}(n)}\right]-\frac{1}{v_{\mathrm{c}}} \frac{\mathrm{d} v_{\mathrm{c}}}{\mathrm{d} n}[n-n(\mu)]
$$

where $n(\mu)$ would be the critical demixing concentration in an experiment at fixed chemical potential and is calculated by derivation of the free energy (28)

$$
[n-n(\mu)]=-\frac{\partial A}{\partial \mu} \sim A_{0}\left[\frac{v-v_{\mathrm{c}}(\mu)}{v_{\mathrm{c}}(\mu)}\right]^{1-\alpha} \frac{\mathrm{d} n}{\mathrm{~d} \mu}\left(\frac{1}{v_{\mathrm{c}}} \frac{\mathrm{d} v_{\mathrm{c}}}{\mathrm{d} n}\right) .
$$

This leads to the following relation between the two scaling variables

$$
\frac{\left[v-v_{\mathrm{c}}(n)\right]-\left[v-v_{\mathrm{c}}(\mu)\right]}{\left[v-v_{\mathrm{c}}(\mu)\right]}=A_{0}\left[\frac{v-v_{\mathrm{c}}(\mu)}{v_{\mathrm{c}}(\mu)}\right]^{-\alpha} \frac{\mathrm{d} n}{\mathrm{~d} \mu}\left(\frac{1}{v_{\mathrm{c}}} \frac{\mathrm{d} v_{\mathrm{c}}}{\mathrm{d} n}\right)^{2} \text {. }
$$

The Fisher renormalization is effective if the difference between the two scaling variables is large i.e. in a temperature range around the critical point of width

$$
\left[\frac{v-v_{\mathrm{c}}}{v_{\mathrm{c}}}\right] \sim\left\{A_{0} \frac{\mathrm{d} n}{\mathrm{~d} \mu}\left(\frac{1}{v_{\mathrm{c}}} \frac{\mathrm{d} v_{\mathrm{c}}}{\mathrm{d} n}\right)^{2}\right\}^{1 / \alpha} \text {. }
$$

The various quantities entering equation (32) are calculated from the free energy (12)

$$
\frac{\mathrm{d} n}{\mathrm{~d} \mu}=\left(\frac{\mathrm{d}^{2} F}{\mathrm{~d} n^{2}}\right)^{-1} \sim \frac{\kappa^{2}}{8 \pi \ell T} .
$$

The critical point is given by the usual Flory result for a polymer in a poor solvent but one must include in $v$ the electrostatic excluded volume

so that

$$
v_{\mathrm{c}}=-\frac{f^{2} 4 \pi \ell}{\kappa^{2}}-\frac{2 w}{N^{1 / 2}}
$$

$$
\frac{\mathrm{d} v_{\mathrm{c}}}{\mathrm{d} n}=\left(\frac{4 \pi \ell}{\kappa^{2}}\right)^{2} f^{2}
$$

The expansion of the free energy around the consolute point shows that

$$
A_{0} \sim T \frac{v_{\mathrm{c}}^{2}}{w^{3} N^{1 / 2}}
$$


The range in the excluded volume parameter $v$ where the Fisher renormalization of the critical exponents is effective is thus

$$
\left[\frac{v-v_{\mathrm{c}}}{v_{\mathrm{c}}}\right]<\left[\frac{4 \pi \ell^{3} f^{4}}{w^{3} N^{1 / 2} \kappa^{6}}\right]^{1 / \alpha}
$$

$\alpha$ is the Ising exponent of the specific heat and has a low value $(\sim 0,11)$ the renormalization of the critical exponents is thus observable if

$$
\kappa^{2} \ll \frac{4 \pi \ell f^{4 / 3}}{w N^{1 / 6}} .
$$

In all cases, this maximum value of $\kappa^{2}$ is smaller than the value $\kappa_{\mathrm{L}}^{2}$ at the Lifshitz point and we thus never expect the Fisher renormalization of critical exponents to be observable. At high ionic strength, the critical exponents of the consolute point are thus the usual Ising exponents.

\section{Mixture between neutral and charged polymers.}

The addition of salt to the polyelectrolyte solution suppresses thus the peak in the polyion structure facture and consequently, the mesophase separation transition becomes a macroscopic demixing transition. An alternative method to destroy the mesophase and to precipitate the polyelectrolyte is to add to the solution a neutral polymer. This process might be of some importance in the understanding of certain biological systems containing weakly charged proteins.

At very low concentration, the neutral polymer is in a poor solvent and thus tends to swell the dense regions of the mesophase. When its concentration $c$ is increased, this is no longer possible and the two polymers phase separate from the solvent. In this first approach, we do not discuss the geometry of the phase diagram neutral polymer-charged polymer-solvent, we only discuss the disappearance of the peak at finite wave vector of the polyelectrolyte structure factor for the simpler case where the neutral polymer has the same chemical nature as the polyelectrolyte.

The Flory free energy of the solution is obtained by generalizing equation (10)

$$
\frac{F}{T}=\frac{\phi}{N} \log \phi+\phi_{\mathrm{i}} \log \phi_{\mathrm{i}}+\frac{c}{P} \log c+\frac{1}{2} v(\phi+c)^{2}+\frac{1}{6} w^{2}(\phi+c)^{3} .
$$

$P$ is the degree of polymerization of the neutral polymer and we have assumed that the non electrostatic interactions are identical between charged and uncharged monomers.

The free energy associated with a fluctuation of the concentration at a wave vector $q$ is

$$
\begin{aligned}
& \frac{\delta F_{q}}{T}=\frac{1}{2}\left\{\delta \phi_{q}^{2}\left[S_{0}^{-1}(q)+v+w^{2}(c+\phi)\right]+\delta \phi_{i q}^{2} \frac{1}{\phi_{\mathrm{i}}}+\delta c_{q}^{2}\left[G_{0}^{-1}(q)+\right.\right. \\
& \left.\left.\quad+v+w^{2}(c+\phi)\right]+2 \delta \phi_{q} \delta c_{q}\left[v+w^{2}(c+\phi)\right]+\frac{4 \pi l}{q^{2}}\left(f \delta \phi_{q}-\delta \phi_{\mathrm{i} q}\right)^{2}\right\} .
\end{aligned}
$$

$G_{0}(q)$ is the Gaussian structure factor of a neutral chain given by the Debye function that we write similarly to that of the polyelectrolyte

$$
G_{0}^{-1}(q)=\frac{1}{P c}\left[1+g\left(\frac{P q^{2} a^{2}}{6}\right)\right] .
$$


Following the same procedure as in section II we calculate the polyelectrolyte-polyelectrolyte correlation function $S(q)=\left\langle\delta \phi_{q} \delta \phi_{q}\right\rangle$

$$
S(q)=\frac{\left(q^{2}+\kappa^{2}\right) A}{\left(q^{2}+\kappa^{2}\right) B+4 \pi \ell f^{2} A}
$$

where we have introduced the notations

$$
\begin{aligned}
& A=G_{0}^{-1}(q)+v+w^{2}(c+\phi) \\
& B=G_{0}^{-1}(q) S_{0}^{-1}(q)+\left[v+w^{2}(c+\phi)\right]\left[G_{0}^{-1}(q)+S_{0}^{-1}(q)\right] .
\end{aligned}
$$

At very low neutral polymer concentration, this structure factor shows a peak at a finite wave vector $q^{*}$ such that

$$
\begin{aligned}
4 \pi \ell f^{2}\left[G_{0}^{-1}\left(q^{*}\right)+v+w^{2}(c+\phi)\right]^{2}= & \left(q^{* 2}+\kappa^{2}\right)^{2}\left\{\frac { g ^ { \prime } a ^ { 2 } } { 6 \phi } \left[G_{0}^{-1}\left(q^{*}\right)+\right.\right. \\
& \left.\left.+v+w^{2}(c+\phi)\right]^{2}+\frac{g^{\prime} a^{2}}{6 c}\left[v+w^{2}(c+\phi)\right]^{2}\right\} .
\end{aligned}
$$

When the neutral polymer concentration vanishes, this gives back the peak position of pure polyelectrolytes (Eq. (14)). As $c$ is increased, the peak position shifts to zero and the structure factor becomes monotonic when

$$
4 \pi \ell f^{2}=\kappa^{4} \frac{g^{\prime} a^{2}}{6}\left[\frac{1}{\phi}+\frac{1}{c} \frac{\left[v+w^{2}(c+\phi)\right]^{2}}{\left[\frac{1}{P c}+v+w^{2}(c+\phi)\right]^{2}}\right]
$$

If the degree of polymerization $P$ is large enough, $\left(P>\frac{1}{f \phi}\right)$ this reduces to

$$
c=4 \pi \ell_{\phi}{ }^{2} \frac{g^{\prime} a^{2}}{6} \text {. }
$$

An extremely small quantity of neutral polymer is thus sufficient to suppress the peak in the structure factor of the polyelectrolyte ; this suggests strongly that only a small quantity of neutral polymer may be dissolved in the mesophase and that a very small quantity of neutral polymer is sufficient to precipitate the polyelectrolyte solution.

\section{Concluding remarks.}

The main result of this paper is that weakly charged polyelectrolytes undergo a mesophase separation transition in a poor solvent that we have studied both at low temperature and in the vicinity of the Flory compensation temperature. The fraction of charged monomers $f$ in the chain could in practice be varied by synthetizing random copolymers between charged and uncharged monomers or by studying polyacids or polybases and adjusting the $\mathrm{pH}$. This would allow a quantitative check of our theoretical results for the phase diagram and the periodicity of the mesophases. Notice however that we have only computed here the stability limit for mesophase separation and not the phase boundaries. The determination of the complete phase diagram and of the symmetry of the phases would require an expansion of the free energy to higher order in the concentration fluctuations (Eq. (11)) at high temperature and a direct comparison of the energies of the phases with various symmetries at low temperature (lamellar phase, hexagonal phase, cubic phase...). 
The effect of the ionic strength is also a strong test of the theory. When salt is added to the solution, the period of the mesophase increases and becomes infinite at high enough ionic strength. The mesophase separation transition becomes then a usual demixing transition. We have discussed in section III the role of the critical fluctuations at the consolute point and at the Lifshitz point associated to the crossover between mesophase separation and demixing. We have not however discussed the role of concentration fluctuations for the mesophase formation. This certainly deserves a detailed study.

An alternative way to decrease the tendency to form mesophases is to add to the polyelectrolyte a small quantity of neutral polymer. We have discussed only very briefly mixtures of neutral and charged polymers in the previous section. The complete phase diagram is certainly rather complex and its determination would be of interest.

Experimentally, the existence of mesophases in polyelectrolyte solutions in poor solvents has been reported in several systems [17] although no quantitative comparison with the present calculations seems possible. Phase transitions have also been studied in great details in polyelectrolyte gels in poor solvents in particular by Tanaka and his coworkers [18]; although our predictions may be affected by the existence of the finite shear modulus of the gel it could be interesting to discuss these experiments along the lines presented here. Some recent scattering experiments of Ilmain, Candau and Schosseler [19] also show a peak in the structure factor of a polyelectrolyte gel (partially neutralized polyacrylic acid) which seems reasonnably well described by equations $(14,15)$.

Finally let us mention the possible extensions of this work to polyelectrolyte solutions in good solvents. The existence and the position of the peak in the structure factor given by equation (14) are independent of the solvent quality and even of the actual functional shape of the free energy chosen to describe the interactions between monomers (that we chose of the Flory form). We expect however a major difference between a poor solvent where the chains are gaussian at short length scales and a good solvent where the chains are locally stretched. One cannot in a good solvent take as a first approximation the Gaussian structure factor as we have done in equation (12). One must introduce the electrostatic persistence length of the chain $\ell_{p}$ and replace in the peak position (14) the monomer size dependence $a^{2}$ by $a \ell_{p}$. The persistence length depends on the ionic strength but also on the monomer concentration; as shown by Witten and Pincus [4], screening by the polymer is a dominant mechanism for semidilute polyelectrolyte solutions in a salt-free good solvent.

\section{Acknowledgements.}

This study was initiated by a discussion with E. Fleury (Rhone-Poulenc Lyon). We also benefitted from useful discusions with M. Cates (Cambridge), P. Pincus (Santa Barbara) and J. Prost (E.S.P.C.I.). We also thank G. Fredrickson (Bell Labs) for mentioning reference (9) while this work almost completed. 


\section{References}

[1] De Gennes P. G., Pincus P., Velasco R. M., Brochard F., J. Phys. France 37 (1976) 461.

[2] Hayter J., Jannink J., Brochard-Wyard F., De Gennes P. G., J. Phys. Lett. France 41 (1980) L451.

[3] ODIJK T., Macromolecules 12 (1979) 686.

[4] Pincus P., Witten T., Europhys. Lett. 3 (1987) 315.

[5] Khokhlov A., Khachaturian K., Polymer 23 (1982) 1742.

[6] Joanny J. F., Pincus P., Polymer 21 (1980) 274.

[7] Кнокнlov A., J. Phys. A A 13 (1980) 979.

[8] LeIbler L., Macromolecules 13 (1980) 1602.

[9] Borue V., ERukhimovich I., Macromolecules 21 (1988) 3240.

[10] De Gennes P. G., Scaling concepts in polymer physics (Cornell University press Ithaca) 1985.

[11] Marques C., Joanny J. F., Leibler L., Macromolecules 21 (1988) 1051.

[12] Landau L., Lifchitz I., Physique statistique (Edition Mir Moscou) 1967, p. 349.

[13] Aharony A., Multicritical points Lecture presented at the advanced course in Theoretical Physics : Critical Phenomena (Stellenbosch) 1982.

[14] Hornkeich R., Luban M., Shtrikman S., Phys. Rev. Lett. 35 (1982) 1678.

[15] Fisher M., Phys. Rev. 176 (1968) 257.

[16] Anisimov M., Voronel A., Gorodetski E., Sov. Phys. JETP 33 (1971) 605.

[17] Huguet J., Vert M., Microdomains in polymer solutions, Ed. Dubin P., Polymer Science and Technology (Plenum) (1987);

STRAUSS U. P., ibid. p. 1.

[18] Tanaka T. et al., Phys. Rev. Lett. 45 (1980) 1636.

[19] Ilmain F., Candau S. J., Schosseler F., in preparation (1989). 